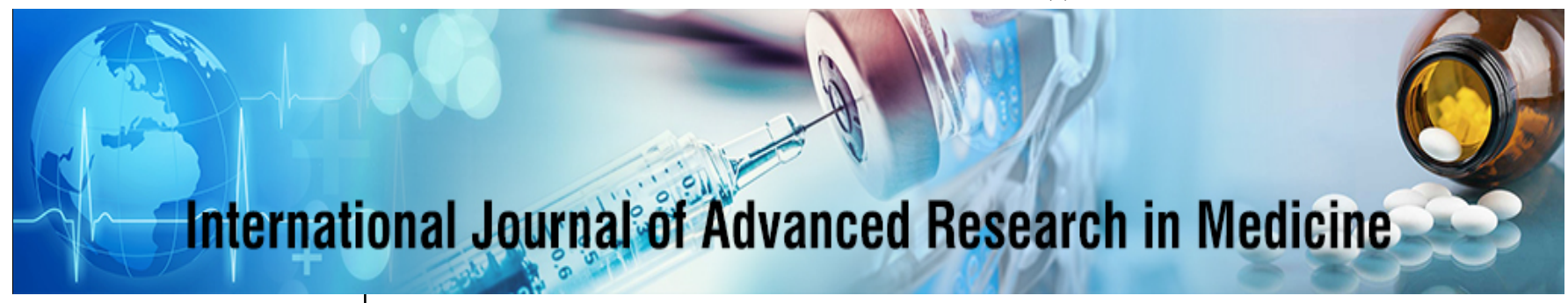

E-ISSN: 2706-9575

P-ISSN: 2706-9567

IJARM 2021; 3(2): 294-299

Received: 22-05-2021

Accepted: 16-06-2021

Dr. Bommakanti Sai Arpita M.B.B.S, Mallareddy Institute of Medical Sciences, Suraram ' $\mathrm{X}$ ' road, Quthubullapur, Hyderabad, Telangana, India

Corresponding Author: Dr. Bommakanti Sai Arpita M.B.B.S, Mallareddy Institute of Medical Sciences, Suraram ' X' road, Quthubullapur, Hyderabad, Telangana, India

\section{Study of lymph node lesions using fine needle aspiration cytology (FNAC)}

\author{
Dr. Bommakanti Sai Arpita \\ DOI: $\underline{\text { https://doi.org/10.22271/27069567.2021.v3.i2e.261 }}$
}

\begin{abstract}
Background: Cytology of lymph nodes has become a window for diagnosis of many diseases. Optimal material and experience, when combined, make cytological diagnosis of equal significance as histopathology. In many clinical settings it is very difficult to decide which patient is more likely to have a reactive or neoplastic lymphadenopathy.

Aim and Objectives: The main aim of the present study to investigate the pattern of lymphadenopathy by FNAC.

Methodology: This retrospective study on aspirates of 50 selected patients with lymphadenopathy, both male and female, was conducted at the Cytology Unit, Pathology Department, Mallaredddy institute of medical sciences, Suraram ' $x$ ' road, Quthubullapur municipality, Hyderabad, Andhra Pradesh from august 2013 to June 2014. This period includes review of literature, data collection, data analysis, discussion and finalizing the report. It is a laboratory investigation based study. Approval of ethics committee was obtained. Lymph node aspirates were collected from these patients using fine needle aspiration cytology (FNAC). These aspirates were then smeared and stained with hematoxylin and eosin and are observed under the microscope.

Results: Out 50 cases, 19 cases (38\%) were non-specific reactive lymphadenitis, 10 (20\%) were tubercular lymphadenitis, $7(14 \%)$ were granulomatous lymphadenitis, 5 (10\%) were metastatic deposits, 4 (8\%) cases were castleman's disease, and there was only 1 case (2\%) of Kikuchi's disease. There were 3 cases (6\%) of Hodgkin's lymphoma and 1 case (2\%) of non-Hodgkin's lymphoma. Acid fast staining was performed in suspected cases of tuberculosis.

Conclusion: Thus, a simple procedure as FNAC was used to identify the pattern of lymphadenopathy, to highlight the incidence of tuberculosis combined with AFB staining, to diagnose malignancies without surgical biopsy and even diagnose rare diseases of the lymph nodes.
\end{abstract}

Keywords: FNAC, lymph nodes, granulomatous lymphadenitis, tubercular lymphadenitis, acid-fast bacilli

\section{Introduction}

A lymph node is an oval shaped organ of the immune system, distributed widely throughout the body and is linked by lymphatic vessels. Lymph nodes act as filters or traps for foreign particles and are important in the proper functioning of the immune system ${ }^{[1]}$. They are packed tightly with white blood cells called lymphocytes. The lymph node consists of dense masses of lymphocyte aggregations intermixed with dilated lymphatic sinus and is supported by a frame work of fine reticular fibres ${ }^{[2]}$. The lymphatic system is structured to address biological infection by other organisms as well as biological breakdowns within the same body. A response to illness that includes inflammation of the lymph nodes specifically indicates a biological factor. Lymph nodes can be diagnosed by biopsy whenever they are inflamed.

\section{Lymphadenitis}

Lymph nodes, the most widely distributed and easily accessible lymphoid tissue, are frequently examined for diagnostic purposes. They are discrete encapsulated structures that contain well-organized B-cell and T-cell zones, which are richly invested with phagocytes and antigen-presenting cells. The activation of resident immune cells leads to morphologic changes in lymph nodes ${ }^{[3]}$.

Nodes involved by acute lymphadenitis are enlarged and painful. When abscess formation is extensive the nodes become fluctuant. The overlying skin is red. Sometimes, supportive infections penetrate through the capsule of the node and track to the skin to produce draining sinuses. Healing of such lesions is associated with scarring. 


\section{Morphology}

Follicular hyperplasia is caused by stimuli that activate humoral immune responses. It is defined by the presence of large oblong germinal centres (secondary follicles), which are surrounded by a collar of small resting naive B cells (the mantle zone) Germinal centres are normally polarized into two distinct regions: (1) a dark zone containing proliferating blast like B cells (Centro blasts) and (2) a light zone composed of $\mathrm{B}$ cells with irregular or cleaved nuclear contours (centrocytes) ${ }^{[4]}$. Interspersed between the germinal $\mathrm{B}$ centres is an inconspicuous network of antigen-presenting follicular dendritic cells and macrophages (often referred to as tingible-body macrophages) containing the nuclear debris of B cells, which undergo apoptosis if they fail to produce an antibody with a high affinity for antigen ${ }^{[4]}$.

\section{FNAC- Fine needle aspiration cytology}

Fine-needle aspiration cytology (FNAC) is a diagnostic procedure used to investigate superficial (just under the skin) lumps or masses. In this technique, a thin, hollow needle is inserted into the mass for sampling of cells that, after being stained, will be examined under a microscope ${ }^{[5]}$. The use of fine needle aspiration cytology (FNAC) in the investigation of lymph node lesions has become an acceptable and widely practised minimally invasive technique, which is safe, simple, rapid and relatively pain-free. It causes minimal trauma to the patient and carries virtually no risk of complications. FNAC can be carried out at the bed-side or in the OPD during the patient's very first visit, without any use of general anaesthetics.

Superficial lymphadenopathy is a common clinical finding; it may be a sign of inflammation, metastatic malignancy or malignant lymphoma. Fine needle aspiration cytology (FNAC) of lymph node has become an integral part of the initial diagnosis and management of patients with lymphadenopathy ${ }^{[6]}$. FNAC is highly cost effective and accurate as a first line investigative technique with differential diagnoses of lymphadenopathy including reactive hyperplasia/inflammatory conditions, granulomatous disorders and malignancy, stratifying cases requiring further investigations, surgical intervention or clinical follow-up ${ }^{[5]}$.

Tuberculous lymphadenitis is a very common cause of superficial lymphadenopathy in countries like India ${ }^{[7]}$. The aim of this study was to describe various cytological pictures of tuberculous lymphadenitis with the irrelative frequency and to assess correlation between FNAC and Ziehl-Neelsen (Z-N) staining in diagnosing tuberculous lymphadenitis.

FNAC is used to assess the staging of primary lymphoid malignancies as well as to recognize the residual, recurrent lymphoid malignancies and also mentioned that the cytology is more readily accepted for the evaluation of deeply seated lymph nodes (i.e. surgically inaccessible) with primary lymphoma or for medically unfit patients for surgery ${ }^{[8]}$. It also provides clues for occult primaries and sometimes also surprises the clinician who does not suspect a malignancy.

Knowledge about the pattern of lymphadenopathy in a population facilitates pathological reporting and helps the clinician in making focused investigation and planning the treatment course. This is a baseline study to investigate the pattern of lymphadenopathy by FNAC.

\section{Aims and Objectives}

1. To determine the spectrum of lesions in lymph node on fine needle aspiration cytology (FNAC).

2. To study the incidence of tuberculosis in lymph node on fine needle aspiration cytology (FNAC).

3. To study the utility of fine needle aspiration cytology (FNAC) in the diagnosis of clinically suspected and unsuspected lymph node malignancy.

4. To compare ultrasound findings with free hand FNAC findings.

\section{Materials and Methods}

This retrospective study on aspirates of 50 selected patients with lymphadenopathy, both male and female, was conducted at the Cytology Unit, Pathology Department, Mallaredddy institute of medical sciences, Suraram ' $x$ ' road, Quthubullapur municipality, Hyderabad, Andhra Pradesh from august 2013 to June 2014. This period includes review of literature, data collection, data analysis, discussion and finalizing the report. It is a laboratory investigation based study.

Institutional Ethics Committee approval was obtained before the initiation of the study. Patients included in the present study were in the age group of 5 years to 80 years. This study was limited to selected patients who were clinically diagnosed with lymphadenopathy for more than three weeks and had undergone FNAC. Patients who have not given consent, Patients suffering from acute lymphadenopathy, those who are suffering from any other illness and pregnant woman were excluded.

In each studied case, a brief clinical history was carried out including age, sex, size, and site of enlarged nodes.

Under aseptic precautions, aspiration of enlarged lymph nodes was performed free hand, using a $23 \mathrm{G}$ needle with 5 cc or 10 cc syringe. The cellular aspirate was spread on a dust-free glass slide and immediately transferred to coplin jars filled with alcohol for fixation. After fixation, slides were stained with Hematoxylin and Eosin (H \& E).

The slides of all cases were examined to determine the cytomorphological features. These features included adequacy, cellularity, arrangement of cells, and nuclear as well as cytoplasmic features. The background was noted in all slides for the presence of necrosis and granuloma formation as well as for the type of inflammatory cells. Hypocellular slides were excluded from the present study. If Tuberculosis (TB) was suspected, slides were also stained with Ziehl-Neelsen methods to detect acid fast bacilli (AFB) directly. Acid fast bacilli (AFB) grading was done on ZiehlNeelsen positive smears. Ultrasound was performed prior to FNAC in all cases. The results expressed as percentage were tabulated.

\section{Results}

Acid-fast bacilli bright red Background blue

Grading of AFB was done as proposed by Kumar et al. ${ }^{[9]}$. Grade 1+ = AFB was found after a careful search Grade 2+ = AFB were singly scattered Grade $3+=$ AFB were found in large numbers arranged in faggots and singly. Bacilli could be detected even under 10 $\mathrm{X}$ magnification. 


\section{Result}

A laboratory investigation based study was carried out using 50 lymph node aspirates, during a period of one year.

Patients with an age range of 5 to 80 years were enrolled for the study. Only 6 cases (12\%) were in the group. 11 patients (22\%) were in range of 18 to 30 years. 15 cases (30\%) belonged to age group between 30 to 50 years and 18 patients (36\%) were above the age of 50 years.

Table 1: Distribution of study population according to their age. $(n=50)$

\begin{tabular}{|c|c|c|}
\hline S.no & Characteristics & No. of patients \\
\hline & Age & $6(12 \%)$ \\
\hline 1 & paediatric age & $11(22 \%)$ \\
\hline 2 & 18 to 30 years & $15(30 \%)$ \\
\hline 3 & 30 to 50 years & $18(36 \%)$ \\
\hline
\end{tabular}

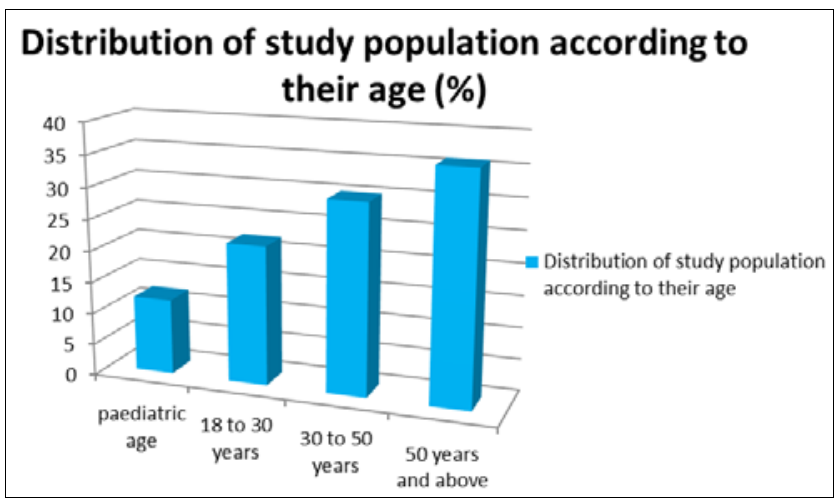

Fig 1: Distribution of study population according to their age

Out of the 50 patients, 27 (54\%) patients were males with 23 (48\%) cases being females leading to an M: F ratio of 1.2:1

Table 2: Distribution of study population according to their sex. $(n=50)$

\begin{tabular}{|c|c|c|}
\hline S. n & Characteristics & No. of patients \\
\hline & Sex & \\
\hline 1 & Male & $27(54 \%)$ \\
\hline 2 & Female & $23(46 \%)$ \\
\hline
\end{tabular}

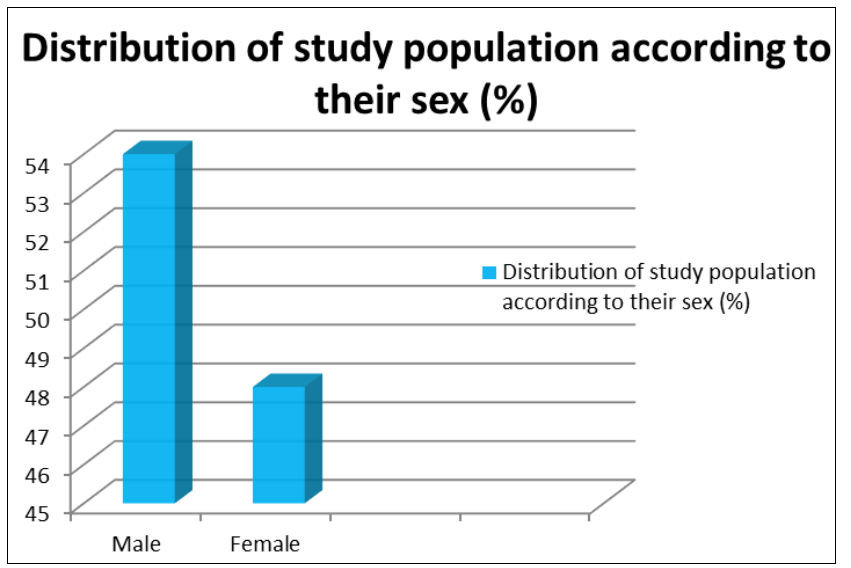

Fig 2: Distribution of study population according to their sex

Cervical lymph node was most commonly involved - 44 cases (88\%). The inguinal lymph nodes were least involved in all types of lymphadenopathy - 1 case (2\%). The remaining cases belonged to axillary group of lymph nodes - 5cases (10\%).

Table 3: Distribution of study population according to the site of lymph node involved. $(\mathrm{n}=50)$

\begin{tabular}{|c|c|c|}
\hline S. n & Characteristics & No. of patients \\
\hline & Site & $44(88 \%)$ \\
\hline 1 & Cervical & $5(10 \%)$ \\
\hline 2 & Axillary & $1(2 \%)$ \\
\hline 3 & Inguinal & \\
\hline
\end{tabular}

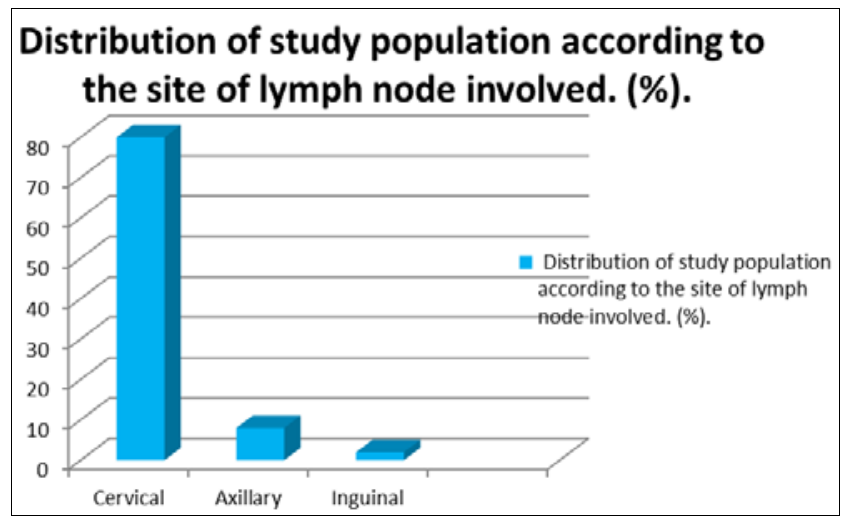

Fig 3: Distribution of study population according to the site of lymph node involved

\section{Ultrasound findings}

Out of 50 cases, 34 cases (68\%) were diagnosed as nonspecific reactive lymphadenitis, 6 cases (12\%) were diagnosed as tubercular lymphadenitis, 5 cases (10\%) were metastatic deposits, and 2 cases (4\%) were castleman's disease, 2 cases (4\%) and 1 cases (2\%) of Hodgkin's and non-Hodgkin's lymphoma were diagnosed respectively.

\section{FNAC findings}

Out of 50 aspirates, FNAC made diagnosis of non-specific reactive lymphadenitis in 19 cases (38\%), 10 cases (20\%) were diagnosed as tubercular lymphadenitis, Granulomatous lymphadenitis was found in 7 cases (14\%), 5 cases (10\%) were metastatic deposits, 1 case (2\%) of Kikuchi's disease and 4 cases (8\%) Castle man's disease was diagnosed and lastly 3 cases (6\%) of Hodgkin's lymphoma and 1 case (2\%) of non- Hodgkin's lymphoma was diagnosed respectively. Out of the 10 cases of tubercular lymphadenopathy, Ziehl Neelsen positivity for acid-fast bacilli was found in 6 cases (60\%).

Table 4: Incidence of Various diseases in aspirated lymph nodes $(\mathrm{n}=50)$

\begin{tabular}{|c|c|c|}
\hline S. n & Characteristics & No. of patients \\
\hline & Diseases & \\
\hline 2 & Non - specific reactive lymphadenitis & $19(38 \%)$ \\
\hline 3 & Tubercular lymphadenitis & $10(20 \%)$ \\
\hline 4 & Granulomatous lymphadenitis & $7(14 \%)$ \\
\hline 5 & Metastasis & $5(10 \%)$ \\
\hline 6 & Castleman's disease & $4(8 \%)$ \\
\hline 7 & Hodgkin's lymphoma & $3(6 \%)$ \\
\hline 8 & Non-Hodgkin's lymphoma & $1(2 \%)$ \\
\hline
\end{tabular}




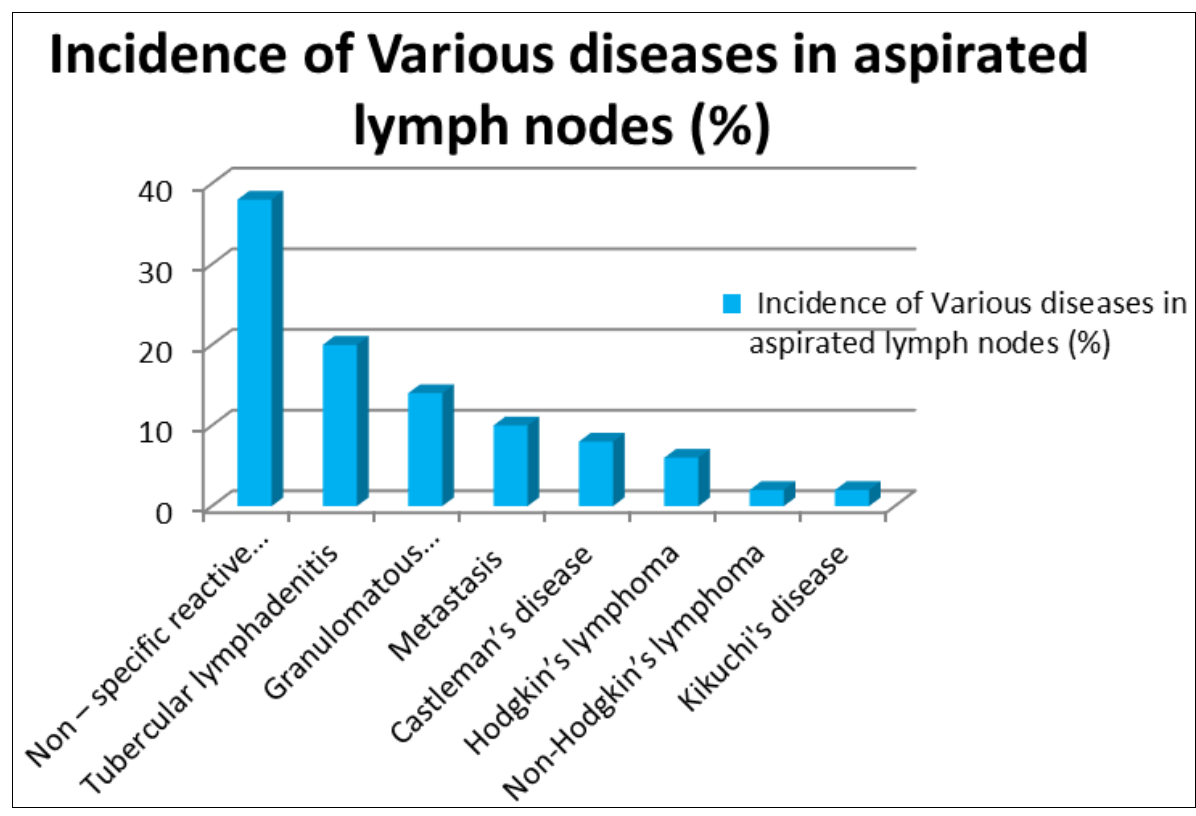

Fig 4: Incidence of various diseases in aspirated lymph nodes

Table 5: Correlation of ultrasound and FNAC findings $(\mathrm{n}=50)$

\begin{tabular}{|c|c|c|c|}
\hline S. n & Disease & No. of cases diagnosed By ultrasound & No. of cases diagnosed by fnac \\
\hline & & & $19(38 \%)$ \\
\hline 2 & Non - specific reactive lymphadenitis & $34(68 \%)$ & $10(20 \%)$ \\
\hline 3 & Tubercular lymphadenitis & $6(12 \%)$ & $7(14 \%)$ \\
\hline 4 & Granulomatous lymphadenitis & ----- & $5(10 \%)$ \\
\hline 5 & Metastasis & $5(10 \%)$ & $4(8 \%)$ \\
\hline 6 & Castleman's disease & $2(4 \%)$ & $3(6 \%)$ \\
\hline 7 & Hodgkin's lymphoma & $1(2 \%)$ & $1(2 \%)$ \\
\hline 8 & Non-Hodgkin's lymphoma & ---- & $1(2 \%)$ \\
\hline
\end{tabular}

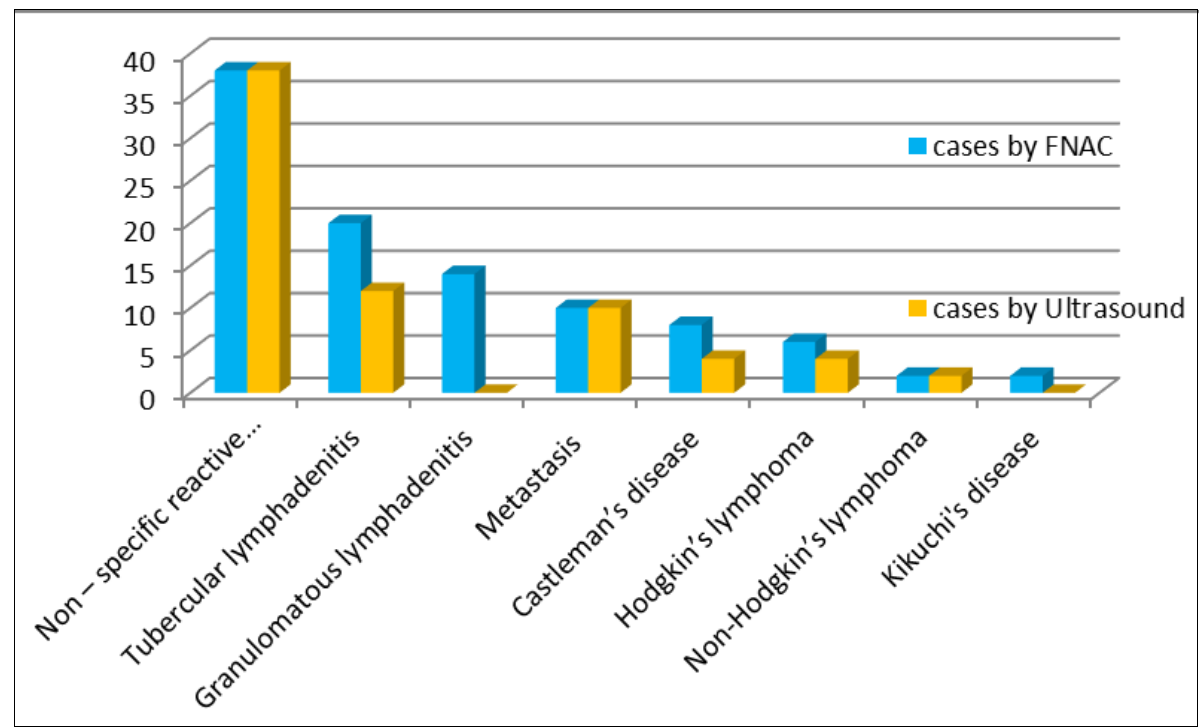

Fig 5: Correlation of ultrasound and FNAC findings (\%)

\section{Discussion}

Cytology of lymph nodes has become a window for diagnosis of many diseases. Optimal material and experience, when combined, make cytological diagnosis of equal significance as histopathology ${ }^{[10]}$. In many clinical settings it is very difficult to decide which patient is more likely to have a reactive or neoplastic lymphadenopathy. Here, knowledge about the pattern of lymphadenopathy is helpful to the clinician for solving the dilemma.
The well-defined role of FNAC in the investigation of lymphadenopathy has previously been studied [11]. In developing countries where facilities for biopsy are not readily available, FNAC is completely safe, quick and inexpensive method for quick diagnosis of lymphadenopathy, reduce need of surgical biopsy.

We have presented our experience with 50 cases of lymphadenopathy over a period of one year. 
Among the 50 cases of lymphadenopathy, maximum number of cases, i.e., 36\% was seen in the age group 50 yrs and above and the least no. of cases, i.e., $12 \%$ were seen in the paediatric age group. This finding is in accordance with study by Hirachand $\mathrm{S}$ et al. in which they also reported a maximum incidence in the ages 50 yrs and above and least cases in the paediatric age group ${ }^{[5]}$. Contrary to this, in study of Bharadwaj $\mathrm{K}$ et al., maximum numbers of cases were seen in 0-10 year's age group ${ }^{[12]}$.

Male to female ratio in our study was 1.2: 1 which matches with the findings of Chawla $\mathrm{N}$ et al. whose study showed a ratio of 1.3: 1 . In comparison to this, females outnumbered males with an M:F ratio of 1:1.05 and 1:1.69, in study of Bharadwaj k et al. and Dasgupta et al., respectively[12],[13]. Lymph nodes of the neck (88\%) followed by axilla (10\%) are the most common sites involved. The site least involved was the inguinal region (2\%). Similar observations have been made in a study by Ruchi Khajuria et al. with Lymph nodes of the neck (83.4\%) followed by axilla(9.6\%) and inguinal $(1.7 \%)^{[14]}$.

In the present series, majority of the cases (38\%) were nonspecfic reactive lymphadenitis. This is in correlation to the findings by Shakya $\mathrm{G}$ et al. where maximum incidence of non-specific reactive lymphadenitis was seen of all the various lesions of the lymph node ${ }^{[15]}$. The aspirate obtained in these cases was greyish in colour. Smears showed polymorphism of lymphocytes, centrocytes, centroblasts, and clusters of histiocytes tangible, body macrophages. There was no evidence of any granulomas or necrosis in the smears studied.

Tuberculosis (20\%) was another important differential diagnostic pattern which is in accordance with the study of Shakya G et al. where $22.4 \%$ cases were tuberculosis ${ }^{[15]}$. Cytosmears show clusters of epitheloid cells mixed with caseous necrotic material, lymphocytes, plasma cells, neutrophils in a dirty necrotic background. FNAC was reported to have $77 \%$ sensitivity in the detection of tubercular lymphadenopathy ${ }^{[16]}$. Out of the 10 cases of tubercular lymphadenopathy, Ziehl Neelsen positivity for acid-fast bacilli was found in 6 cases (60\%).This is in correlation with the study conducted by Lakhey $\mathrm{M}$ et al. ${ }^{[18]}$ where Ziehl Neelsen positivity was $58.1 \%$.

Out of the 50 aspirates Granulomatous lymphadenitis was identified in 7 aspirates (14\%), which is close to the findings of Shakya $G$ et ${ }^{[15]}$. al where $12 \%$ granulomatous lymphadenitis was diagnosed. Aspirate in these cases was blood mixed and smears showed lymphocytes, plasma cells, histiocytes and epitheloid cell clusters in a hemorrhagic background.

Metastatic deposits were reported in $10 \%$ of the cases. Where $10.7 \%$ cases were metastatic deposits. Aspirates of metastases were blood mixed. Smears showed numerous singly dispersed clusters and sheets of pleomorphic cells, with pleomorphic hyperchromatic nuclei. Background shows inflammatory cells, necrotic debris and blood elements.

Incidence of Hodgkin's and non - Hodgkin's lymphoma was $6 \%$ and $2 \%$ respectively in accordance to a study conducted by V Koo et al. ${ }^{[17]}$. Smear shows polymorphous population of cells comprising of lymphocytes, eosinophils, occasional plasma cells and large mono-nucleate and binucleate pleomorphic Reed-Sternberg (RS) cells in a hemorrhagic background in Hodgkin's Lymphoma.

$8 \%$ of the cases were diagnosed as castleman's disease. Smears were highly cellular and showed polymorphous cell population with predominance of small lymphocytes and few tingible-body macrophages. Few singly scattered large oval cells with indistinct margins were seen. These cells had pale cytoplasm with fine chromatin with inconspicuous nucleoli. There was prominent vascularity with capillaries. There were no epithelioid cells, giant cells or necrosis. However, few immunoblasts were seen. Plasma cells were few in number.

Kikuchi-Fujimoto's disease (KFD) or histiocytic necrotizing lymphadenitis is an uncommon, benign, self-limited disease of unknown etiology, primarily affecting young women ${ }^{[19]}$. $2 \%$ of the cases belonged to this group. Smears showed polymorphous lymphoid population intermingled with histiocytes, many of which showed a small size, eccentric location, crescent-shaped nuclei and abundant karyorrhectic debris in the background.

\section{Conclusion}

Fine Needle Aspiration Cytology is a simple, safe, rapid, cost effective and reasonably accurate method of establishing the diagnosis of lymphadenopathy. FNAC is a very useful and sensitive diagnostic aid in patients with lymphadenopathy and should be the first investigation to be performed when patients complain of lymph node enlargement wherever in the body. Fine Needle Aspiration Cytology is a valuable tool for identification of opportunistic infections, neoplastic lesions and nonneoplastic lesions. We recommend that Fine Needle Aspiration Cytology should be the first diagnostic procedure in clinics for the diagnosis of the cause of lymphadenopathy in patients, because it is practical, convenient, safe, and relatively painless, does not require hospitalization or anesthesia does not leave a scar and gives a rapid result for initial diagnosis than conventional biopsy. Combination of the needle aspiration cytology with acid fast staining is highly valuable for routine diagnosis of tuberculosis. Other ancillary investigations like mycobacterial culture, lymph node biopsies and polymerase chain reaction can be reserved for cases, in which there is a strong clinical suspicion with equivocal result of FNAC and acid-fast staining. FNAC can also be used to detect rare disease like Kikuchi's and Castleman's disease.

\section{Acknowledgment}

The author is thankful to Department of Pathology for providing all the facilities to carry put this work.

\section{Conflict of Interest \\ None}

\section{Funding Support}

Nil

\section{References}

1. Swollen Glands and Other Lumps under the Skin-Topic Overview. Children.webmd.com 2011-04-14. Retrieved 2014-02-28.

2. Kaldjian Eric P, Elizabeth Gretz J, Arthur Anderson O, Yinghui Shi, Stephen Shaw. Spatial and molecular organization of lymph node T cell cortex: a labyrinthine cavity bounded by an epithelium-like monolayer of fibroblastic reticular cells anchored to basement membrane-like extracellular matrix". International Immunology (OxfordJournals) 2001;13(10):12431253. 
Doi: $\quad$ 10.1093/intimm/13.10.1243. PMID 11581169.

Retrieved 2008-07-11

3. Molander DW. Diseases of the Lymphatic systemDiagnosis and Therapy 1983.

4. Robbins And Cotran Pathologic Basis of Disease, 7th Edition, Vinay Kumar, Mbbs, Md, FRC Path; Abul K. Abbas, MBBS; Nelson Fausto, MD, Elsevier 2005. Imprint: W.B. Saunders Company. ISBN: 0-7216-01871.

5. Hirachand S, Lakhey M, Akhter J, Thapa B. Evaluation of fine needle aspiration cytology of lymph nodes in Kathmandu Medical College, Teaching hospital. Kathmandu Univ Med J 2009;7(26):139-142.

6. Keith VE, Harsharan SK, Jerald GZ. Fine needle aspiration biopsy of lymph nodes in the modern era: reactive lymphadenopathies. Pathol Case Rev 2007;12(1):27-35.

7. Mohapatra PR, Janmeja AK. Tuberculous lymphadenitis. J Assoc Physicians India 2009;57:58590.

8. Alam K, Khan A, Siddiqui F, Jain A, Haider N, Maheshwari V. Fine needle aspiration cytology (FNAC): A handy tool for metastatic lymphadenopathy. Int J Pathol 2010;10:2.

9. Kumar S, Ferns S, Sujatha S, Jatiya L. Acid-fast staining patterns and their correlation with HIV positivity (lett). Acta Cytol 2005;49:111-2.

10. Koss LG. Diagnostic Cytology and the Histopathological Basis. 4thed. Philadelphia: Lippincott Company 1994, 194-8.

11. Steel BL, Schwart MR, Ramzy I. Fine needle aspiration biopsy in the diagnosis of lymphadenopathy in 1103 patients. Role, limitations and analysis of diagnostic pitfalls. Acta Cytol 1995;39(1):76-81.

12. Bharadwaj K, Bharadwaj BI, Goyal T. FNAC in lymph node disorders with special reference to tuberculosis. J Cytol 2000;17:155-159.

13. Dasgupta A, Gosh RN, Poddar AK et al. FNAC of cervical lymphadenopathy with special reference to tuberculosis. J Indian Med Assoc 1994;92:44-46.

14. Ruchi Khajuria, Goswami KC, Singh K, Dubey VK. Pattern of Lymphadenopathy on Fine Needle Aspiration Cytology in Jammu. JK science 2006, 8(3).

15. Shakya G, Malla S, Shakya KN, Shrestha R. A Study of FNAC of Cervical Lymph Nodes, J Nepal Health Res Counc 2009;7(14):1-5.

16. Lau SK, Wei WI, Hsu C, Engzell UCG. Efficacy of fine needle aspiration cytology in the diagnosis of tuberculous cervical lymphadenopathy. The Journal of Larynology and Otology 1990;104:24-9.

17. Koo V, Lioe TF. RAJ Spence Fine needle aspiration cytology (FNAC) in the diagnosis of granulomatous lymphadenitis, Ulster Med J 2006;75(1):59-65.

18. Lakhey M, Bhatta CP, Mishra S. Diagnosis of Tubercular Lymphadenopathy by Fine Needle Aspiration Cytology, Acid-Fast Staining and Mantoux test, J Nepal Med Assoc 2009;48(175):230-33.

19. Sivakumar S, Ramamurthy fine needle aspiration cytology of kikuchi-fujimoto's disease: a report of 2 cases with emphasis on cytologic features and differential diagnosis. Acta Cytol 2012;56(4):457-62. 